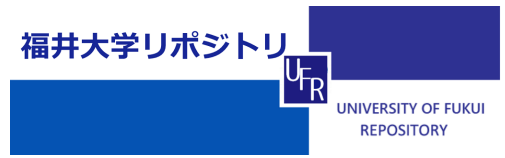

Mdel I ing and si mul at i on of gyr ot rons

\begin{tabular}{|l|l|}
\hline 著者 & $\begin{array}{l}\text { SABCHEVSK S, I DEHARA T, GLYAVI N M OGAWA I , } \\
\text { M TSUDO S }\end{array}$ \\
\hline $\begin{array}{l}\text { j our nal or } \\
\text { publ i cat i on t i t I e }\end{array}$ & $\begin{array}{l}\text { Vacuum - Sur f ace Engi neer i ng Sur f ace } \\
\text { I nst r unent at i on and Vacuum Technol ogy }\end{array}$ \\
\hline vol une & 77 \\
\hline number & 4 \\
\hline page r ange & $519-525$ \\
\hline year & $2005-03$ \\
\hline URL & ht t p: //hdl . handl e. net /10098/1607 \\
\hline
\end{tabular}




\title{
Modelling and simulation of gyrotrons
}

\author{
S. Sabchevski ${ }^{\mathrm{a}}$, T. Idehara ${ }^{\mathrm{b}}$, M. Glyavin ${ }^{\mathrm{c}}$, I. Ogawa ${ }^{\mathrm{d}}$, S. Mitsudo \\ anstitute of Electronics, Bulgarian Academy of Sciences, Sofia 1784, Bulgaria \\ ${ }^{b}$ Research Center for Development of Far-Infrared Region, Fukui University, Fukui 910-8507, Japan \\ 'Institute of Applied Physics, Russian Academy of Sciences, N. Novgorod 603950, Russia \\ ${ }^{\mathrm{d}}$ Cryogenic Laboratory, Faculty of Engineering, Fukui University, Fukui 910-8507, Japan
}

\begin{abstract}
Modelling and simulation of gyrotrons have two interconnected aspects, namely beam formation in the electronoptical system (EOS) and beam-field interaction in the resonant cavity. In this paper we address both problems and outline the physical models and numerical techniques implemented in our problem-oriented package of computer codes. In order to illustrate our approach we present some results of numerical experiments carried out at the FIR FU centre and directed towards analysis and optimization of the existing devices of the Gyrotron FU Series as well as applied to the development of simulation-based design (SBD) of a novel high harmonic gyrotron with the electron beam encircling the axis and a permanent magnet system.
\end{abstract}

Keywords: Gyrotron; Modelling; Simulation-based design (SBD)

\section{Introduction}

Modelling and simulation are the two indispensable components of the paradigm of computer-aided design (CAD) based on numerical experiments. Nowadays, CAD is a widely used technique for engineering and optimization of various electron devices including the gyrotrons as one specific class of them (see for example [1-5]). Moreover, it gives deeper physical insight into their operational performance and allows one to study and predict basic characteristics of the tubes in a broad parameter space in a more economical, flexible and informative way compared with direct measurements. Over the years a great number of researchers and institutions have been involved in the development of physical models and computer codes for simulation of different gyro-devices worldwide [1-5]. Their limited availability and narrow specialization for the solution of specific problems, however, continue to motivate many groups to develop their own simulation tools. Aiming for further 
development of the Gyrotron FU Series and optimization of the existing devices, similar work is being carried out in the FIR Research centre FU (Fukui, Japan) in collaboration with IE-BAS (Sona, Bulgaria) and IAP.RAS (N. Novgorod, Russia). Its main goal is the development of erfective sofware packages based on adequate physical models and characterized by efective program implementation. In this paper, we outine and illustrate with specific examples some of the developed codes. These are: (i) GUN-MIG/CUSP software package for CAD of electron-optical systems (EOS) of gyro-devices; (ii) cavity simulation-time independent single mode code (CS. TISM) and (iii) cavity simulation-mult mode code $(\mathrm{CS}-\mathrm{MM})$.

\section{Ray racing software package GUN-MTG/CUSP}

GUN-MIG/CUSP is a problem-oriented trajectory analysis tool that traces the electron trajectories in combined static axisymmetric electric and magnetic felds and calculates beam parameters and electron-optical characteristics of the electron gun [6,7]. Both, magnetron injection guns and guns with field reversal (or cusp guns) can be simulated. The software package is based on a 2.5 dimensional (2-1/2D) self-consistent physical model which comprises of relativistic equations of motion, Poisson's equation and relations goveming extraction of beam current from different cathode regions (virtual planar diodes with thermionic emitters) in an axially symmetric EOS. A square-shaped grid and a fve-point discretization formula are used for the solution of the boundaryvalue problem by the finite difference method (FDM) with successive over-relaxation (SOR) on a sequence of three grids with increasing fnesse (i.e. decreasing step). The potentials and fields are interpolated using a 4 by 4 mesh points stencil for Lagrange interpolation. The axial magnetic field is input as a set of polynomial expansion coefficients or directly as an array produced by a separate program for magnet design. The off-axis components of the magnetic field are computed from the paraxial expansion. The electrons of the beam contribute to the electric feld through their space charge. A finite number of electron rajectories, each associated with a definite current density are traced through the system integrating their equations of motion by the fourth-order Runge-Kutia method. The space-charge distribution is computed using a combination of the particle-in-cell method with the area-weighted algorithm for allocation of the charges to the mesh. The cells are defined by the same mesh, which is used for the solution of the boundary value problem. For each time step inside a given cell, the charge is deposited to the four adjacent vortices of the cell (mesh nodes) according to the area-weighted algorithm. Allocating the charge and summing up the contribution of all rays the charge density in each node is obtained. The region near the emitter is divided into a number of small virtual diodes in which the current is governed by the potential distribution and the initial velocities of the themoelectrons. It is also assumed that in each virtual diode the Langmuir theory holds and the technique implemented in GUN-EBT sofware package [8] is applied for the computation of the extracted currents.

GUN-MIG/CUSP is written in FORTRAN-77 and is operational on an IBM compatible PC. It consists of a set of computational modules and posi-processor program intended for systematization and visualization of the results of numerical experiments. Information is presented in a sequence of plots and tables on seven screens altogether and is stored in fles with generic names, which refect the type of data they contain and the variant of the analyzed system. In such a way, by running the code with different initial parameters and conditions, a database containing well-structured results from various simulations is being created.

A typical triode magnetron injection gun (MIG) analyzed using GUN-MIG/CUSP is shown in Fig. I together with the results of trajectory analysis. A series of such guns, which differ in the configuration, sizes and positions of the electrodes have been studied [6].

The outlined software package has also been used for computer-aided electron-optical design of a novel EOS for a high hamonic gyrotron with an 
axis-encircling electron beam and magnetic system utilizing a permanent magnetic circuit [7]. A distinguishing feature of the novel EOS is the use of a gradual field reversal instead of an abrupt one as in the traditional cusp guns. The configuration of the electron gun together with the axial magnetic feld distribution and electron trajectories are shown in Fig. 2. The required magnetic field is produced by a system which utilizes a permanent rare earth (NdFeB) magnetic circuit, designed and manufactured by the Shin-Etsu Chemical Co. using the ELF/MAGIC code [9]. It

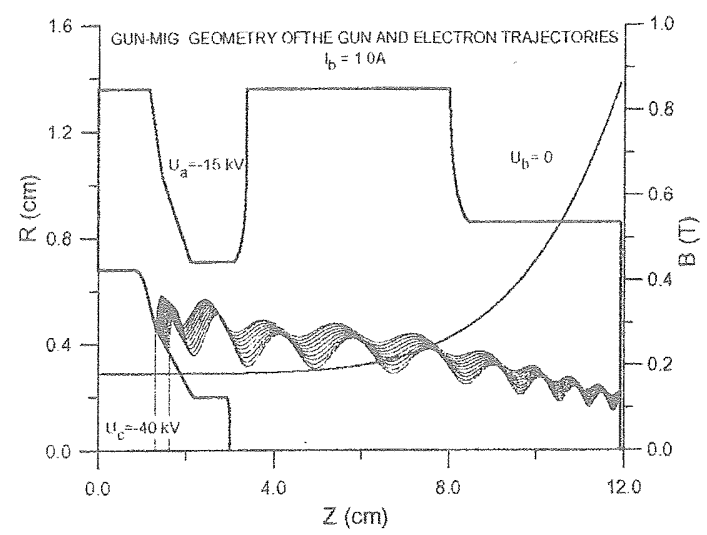

Fig. 1. Magnetic feld distribution and electron orbits in a triode magnetron injection gun. is of a hybrid type and is composed of rings with magnetization oriented radially and axially so arranged as to produce an appropriate field profile having a long enough flattop region along the resonant cavity. For fine tuning of the magnetic field distribution a set of adjusting bolts as well as additional coils are used. The final configuration of the EOS has been optimized after a great number of iterations and is characterized by a high electron-optical performance. High-quality electron beams can be produced with adequate velocity ratio, small velocity spread and low ripple. Based on the computer-aided electron-optical design the mechanical design of the EOS has been performed. The main parts of the tube have been manufactured by GYCOM (N. Novgorod, Russia). The whole device has been assembled and put in operation successfully at the FIR FU Research Centre. Preliminary experiments with the novel gyroton have confirmed the high electron-optical performance of the EOS as predicted by numerical simulations.

\section{Simgle mode time indenendent code CS-TISM}

The physical model realized in CS-TISM code is one, originally proposed by Flifet et al. [10]. In accordance with this model, the electron beam is

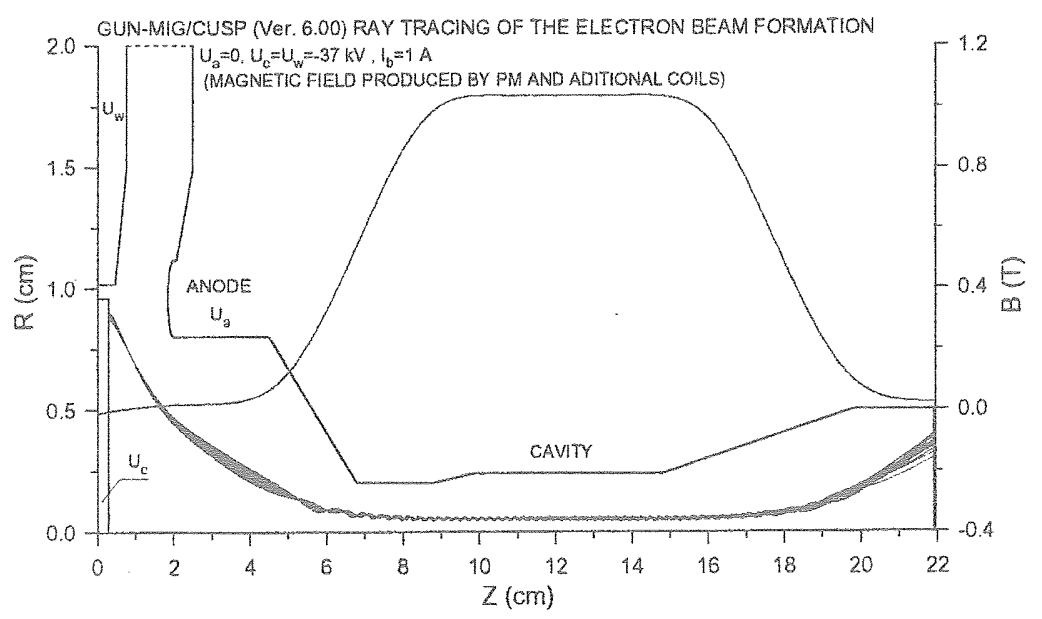

Fig. 2. Consiguration of the EOS for LOG, magnetic field profile and electron trajectories. 
represented as an ensemble of particles and their trajectories are traced in an ambient magnetic field $\vec{B}_{0}=B_{0} \vec{z}$ and high-requency electric feld $\vec{E}=$ $E_{y} \vec{F}+E_{\theta} \vec{\theta}$ of a $T E_{m n}$ mode in a cylindrical coordinate system with unit vectors $\vec{\gamma}, \vec{\theta}$ and $\vec{z}$. The field components are given by

$E_{r}(r, \theta, z, l)=\left(\vec{z} \times \nabla \psi_{m n}\right)_{r} F_{m n}(z) \exp (i \omega t)$,

$\mathbb{E}_{\theta}(r, \theta, z, t)=\left(Z \times \nabla \psi_{m n}\right)_{\theta} F_{m n}(z) \exp (\mathrm{i} \omega t)$,

where the membrane function $\psi_{m n}=$ $C_{m n} J_{m}\left(k_{m n} l\right) \exp (-\mathrm{im} \theta)$ is a solution of the corresponding wave equation for a cylindrical waveguide. Here $J_{m}(x)$ is the $m$ th order Bessel function of the first kind and the transverse wave number $k_{m n}=\chi_{m n} / R_{c a v}$ is determined by the boundary condition $\mathbb{J}_{m}^{\prime}\left(\chi_{m n}\right)=0$ on the cavity wall of radius $R_{\text {cav }}$. The nomalization coefficient is given by $C_{m n}=\left[\sqrt{\pi\left(\chi_{m n}^{2}-n^{2}\right)} J_{m}\left(\chi_{m n}\right)\right]^{-1}$. The axial dependence of the field intensity is described by the complex profile function $F_{m n}(z)=$ $F(z) \exp [-i \phi(z)]$ with amplitude $F(z)$ and phase $\Phi(z)$. As usual, hamonic time dependence with an angular frequency $\omega$ of all field components is assumed. It is presumed also that the rotation of the $\mathrm{TE}_{m n}$ modes is in the same direction as the gyromotion of the electrons (co-rotation). A mimus sign in the exponent, which depends on the azimuthal angle, corresponds to the co-rotation. The individual electron orbits are described by the relativistic equation of motion

$m_{0} \frac{\mathrm{d} \vec{p}}{\mathrm{~d} t}=-e\left(\vec{E}+\frac{\vec{p}}{\gamma} \times \vec{B}_{0}\right)$

where $\vec{p}=\gamma \vec{v}$ is the nomalized momentum expressed by the velocity of the electron $\vec{v}$ and the relativistic factor $\gamma=\left(1+p^{2} / c^{2}\right)^{1 / 2}$. Here $m_{0}$ is the rest mass of an electron and $c$ is the speed of light. In a slow-time-scale formulation Eq. (3) is reduced to the following two equations for the normalized transverse momentum $p_{2}$ and the slow time scale variable $A$

$\frac{d p_{t}}{d t}=-A_{m n}\left(s, R_{g c}\right) B_{m n}^{(-)}\left(s, R_{L}\left(p_{l}\right)\right) F(z) \cos (s \Lambda-\psi)$,

$$
\begin{aligned}
\frac{\mathrm{d} A}{\mathrm{~d} r}= & \frac{1}{p_{1}} A_{m n}\left(s, R_{g c}\right) B_{m n}^{(+)}\left(s, R_{I}\left(p_{l}\right)\right) \sin (s \Lambda-\Psi) \\
& +\frac{\omega}{s}-\frac{R_{0}}{\gamma}
\end{aligned}
$$

where $s$ is the harmonic number, $R_{g c}$ is the radius of the guiding centre of the electron orbit, $R_{L}=$ $p_{t} / \Omega_{0}$ is the Lamor radius, $\Omega_{0}=e B_{0} / m_{0}$ is the cyclotron frequency and $A_{m n}\left(s, R_{g c}\right)=k_{m n} C_{m n}$ $J_{m-s}\left(k_{m n} R_{g c}\right), \quad B_{m m}^{( \pm)}\left(s, R_{L}\right)=\frac{e}{2 m_{0}}\left[J_{s-1}\left(k_{m n} R_{L}\left(p_{t}\right)\right) \pm\right.$ $\left.J_{s+3}\left(k_{m n} R_{I}\left(p_{t}\right)\right)\right]$.

The axial dependence of the field intensity is described by an equation which follows from the theory of weakly irregular waveguides [11]

$$
\begin{aligned}
& \frac{\mathrm{d}^{2} F_{m n}}{\mathrm{~d} z^{2}}+k_{z}^{2} F_{m n} \\
& =-i \mu \omega I_{b} A_{m n}\left(s, R_{g c}\right) \\
& \quad \times \frac{1}{2 \pi} \int_{0}^{2 \pi} a J_{s-1}\left(\frac{k_{m n} p_{l}}{\Omega_{0}}\right) \exp (-\mathrm{i} s \Lambda) \mathrm{d} \Lambda_{0}
\end{aligned}
$$

where $l_{b}$ is the total beam current, $\alpha=v_{i} / v_{z}$ is the orbital-to-axial velocity ratio (pitch factor). Here the longitudinal wave number $k_{z}=\left[\omega^{2} / c^{2}-\right.$ $\left.\chi_{m n}^{2} / R^{2}(z)\right]^{1 / 2}$ depends on the axial coordinate through the cavity radius $R(z)$. If the cavity radius changes slowly along the axis we can consider a weakly irregular waveguide as one where the fields at any $z$ have one and the same transverse structure and difer only in their amplitude through the function $F_{m n}(3)$. Indeed, the gyrotron interaction cavities are typically cylindrical, with tapers of various sizes and lengths on either end to aid in mode selection and to improve the efficiency of interaction. Such cavities are capable of supporting a large number of modes, but gyrotrons are typically operated near cut-orf $\left(k_{m n} \gg k_{z}\right)$. Therefore, at the gun end the waveguide is well and truly cutoof. The wave there is evanescent and its amplitude becomes smaller the closer it goes towards the electron gun. On the contrary, at the output end there is an outgoing wave travelling in the $z$-direction. This situation is described by the following somcalled radiation boundary conditions

$\frac{d F_{m u}(z)}{d z} \mp i k_{2} F_{m n}(z)=0$ 
where the minus sign corresponds to the entrance $\left(z=z_{\text {in }}\right)$ and the plus to the exit $\left(z=z_{\text {out }}\right)$ end of the cavity.

Calculation of the effciency involves an average over all the electrons in the beam. The electronic efficiency (efficiency of the interaction between beam electrons and the field) is given by

$\eta_{e l}=\left\langle\frac{\gamma_{0}-\gamma_{i}}{\gamma_{0}-1}\right\rangle$

where $\gamma_{0}$ and $\gamma_{i}$ are initial and final values for the electron, respectively, and the average $\langle$ is taken over the ensemble of particles representing the beam.

The outlined code has been used for simulation of a gyrotron with an electron beam encircling the axis. An inherent feature of such a device is its high-hamonic operation due to the fact that an axis encircling beam couples effectively only with co-rotating modes having azimuthal indices equal to the resonant hamonic number. This leads to greater mode separation and eventually to greater mode selectivity. As the harmonic numbers for such scheme of operation can be significantly greater compared with traditional gyrotrons, the reguired magnetic field for gyro-devices with axisencircling beams is greatly reduced and can be produced by a pemanent magnet, thereby eliminating the need for a cryogenic superconducting magnet. An important advantage of the gyro-

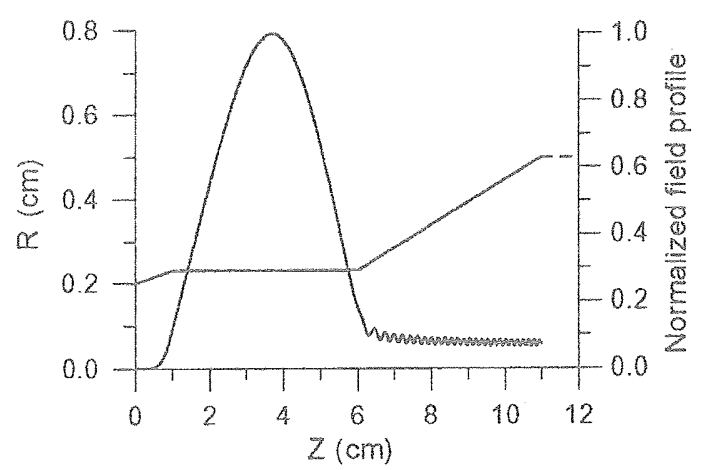

Fig. 3. Configuration of the resonant cavity and longitudinal field profile (accelerating voltage $U_{a}=37 \mathrm{kV}$, beam current $I_{b}=1.5 \mathrm{~A}$, average velocity ratio $\alpha=1.6$, beam ripole $A_{r}=8 \%$, maximum magnetic field at the llat-top region $B_{0}=1.06 \mathrm{~T}$, frequency mismatch $\delta=0.54 \%$ ).

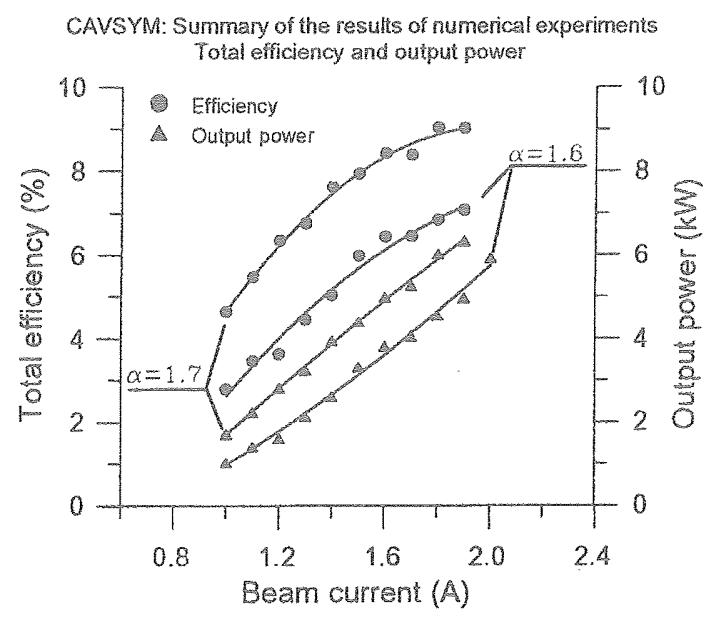

Fig. 4. Total efficiency and output power vs. beam current for two values of the velocity ratio.

devices with permanent magnets is that their weight and sizes are considerably reduced. Additionally, such portable devices are more economical and simple in operation. The absence of mode competition which is a severe problem for the conventional gyrotrons, especially at higher harmonics) in LOG guarantees a single mode operation. For such situation, the single-mode physical model described above and implemented in the CS-TISM code is an adequate choice. As an ilustration we present, here, the results from the calculation of the total efficiency of the beam-field interaction in a resonant cavity optimized for operation at fourth hamonic of the $T E_{41}$ mode. The configuration of the resonator and the longitudinal field profile in it are presented on Fig. 3. The dependence of both the total efficiency and the output power on beam current is shown in Fig. 4 for two values of the average velocity ratio of the electron beam. The first experimental results obtained after the tube was put into operation corroborate the predictions of the numerical experiments.

\section{Nultimode code CS-MM}

In the CS-MM code a well known and widely used multimode physical model is realized (see, 
e.g. $[12,13]$ ). It consists of a system of equations that describe self-consistenty both the dynamics of electrons and excitation of the resonant cavity. Representing the high-rrequency field as a superposition of modes labelled by the index $s$ the equation of electron motion takes the form

$$
\begin{aligned}
& \frac{d p}{d \zeta}-i\left(\Delta+|p|^{2}-1\right) p \\
& =i\left(\sum_{s} p^{n_{s}-1} F_{s} f_{s}(b) \exp \left[\left(\Psi_{s}-n_{s} 0_{0}\right)\right]\right)^{*},
\end{aligned}
$$

where $p=\left(p_{i} / p_{t 0}\right) \exp \left[-\mathrm{i}\left(\beta-\beta_{0}\right)\right]$ is the nomalized orbital momentum, $\theta_{0}$ is the initial value of the slowly varying phase $\theta=\theta-\omega_{a}, \theta$ is the azimuthal coordinate of the electron in the frame of the guiding centre, $\omega_{a}$ is the frequency over which averaging is performed, $\Delta$ is the normalized freguency mismatch and $\zeta$ is the nomalized axial coordinate. Here $n_{s}$ is the hamonic number of $s$ th mode with longitudinal profle $f_{s}(\zeta)$. The nomal. ized mode amplitudes $F_{S}$ and phases $\Psi_{s}$ obey the system

$\frac{\mathrm{d} F_{s}}{\mathrm{~d} \tau}=F_{s}\left(-\frac{n_{s}}{2 Q_{s}}+\Phi_{s}^{\prime}\right), \quad \frac{\mathrm{d} \Psi_{s}}{\mathrm{~d} t}=\frac{\omega_{s}^{\prime}}{\omega_{a}}-n_{s}+\mathscr{\Phi}_{s}^{\prime \prime}$,

where $\tau=\omega_{a} t$ is the dimensionless time, $\omega_{s}=\omega_{s}^{\prime}+i \omega_{s}^{\prime \prime}, \quad \omega_{s}^{\prime \prime}=\omega_{s}^{\prime} / 2 Q_{s}, Q_{s}$ is the quality Aactor. The complex value $\Phi_{s}=\mathscr{W}_{s}^{\prime}+i \phi_{s}^{\prime \prime}$ which characterizes interaction between the electron beam and sth mode, is given by

$$
\begin{aligned}
\Phi_{s}= & -i \frac{H_{s}}{F_{s}} \frac{1}{2 \pi} \int_{0}^{2 \pi}\left[\frac { 1 } { 2 \pi } \int _ { 0 } ^ { 2 \pi } \left(\int_{0}^{\zeta_{o u t}}\left(p^{*}\right)^{n_{s}} f_{s}^{*}(\zeta)\right.\right. \\
& \left.\left.\times \exp \left[-\mathrm{i}\left(\Psi_{s}-n_{s} g_{0}\right)\right] \mathrm{d} \zeta\right) \mathrm{d} 8_{0}\right] \mathrm{d} \phi,
\end{aligned}
$$

where $\mathbb{l}_{s}$ is the dimensionless current $[12,13]$.

The code CS-MM exists in two versions, namely for a PC (Fortran 77) and an SK-5 computer (Fortran 90).

As illustrative example demonstrating the capabilities of the CS-MM code we present, here, some results from numerical experiments carried out for the investigation of the interaction between $\mathrm{TE}_{23}$ (at fundamental cyclotron resonance) and $\mathrm{TE}_{26}$ (at the second cyclotron hamonic) modes in the FU-II Gyrotron. Depending on the opera. tional parameters of the tube (beam current $I_{b}$ accelerating voltage $U_{b}$, velocity ratio $A$ LFA and magnetic field $B$ or frequency mismatch DEL TA) several different regimes can be observed such as single mode excitation, mode suppression, mode competition and mode cooperation. The temporal
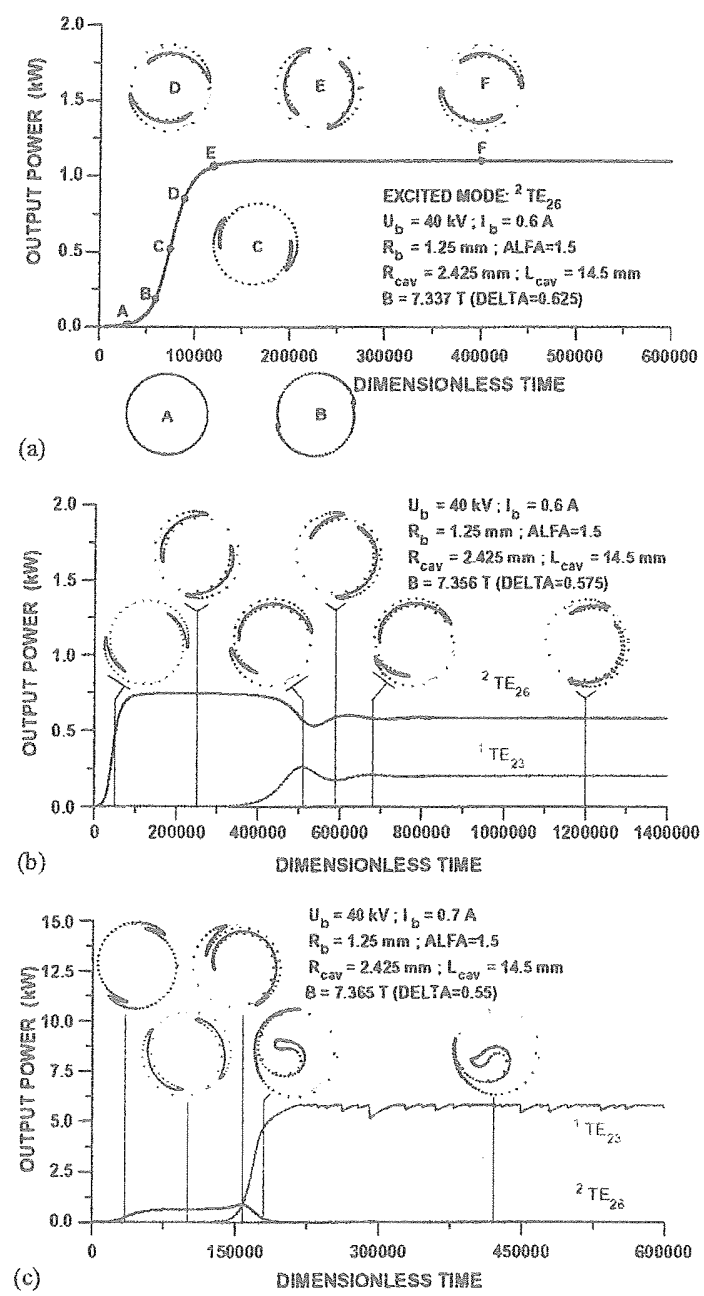

Fig. 5. Temporal evolution of mode excitation and bunching of the electrons: (a) single mode operation at second harmonic, (b) mode competition between $T_{23}$ and $T_{269}$ (c) mode cooperation ( $\mathrm{TE}_{26}$ mode facilitates the excitation of $\mathrm{TE}_{23}$ mode). The basic parameters of the electron beam (voltage $U_{b}$, curtent $I_{b}$ and velocity ratio ALFA), resonant cavity (radius $R_{\text {can }}$ length $\left.L_{\text {cau }}\right)$, magnetic field $B$ and rrequency mismatch DELTA are given in the insets of the diagrams. 
evolution of the bunching process and mode dynamics in several characteristic cases are presented in Fig. 5.

\section{Conclesions}

The computer codes outlined form the core of the developed software package. This package also includes several supplementary modules for preand post-processing of the simulation data. A distinguished and advantageous feature of this set of programs (in comparison with other known tools) is that they are united by a common functional assignment and are stored in a wellstructured library which can easily be extended by the addition of new modules. In such a way the software package allows one to perform a complete analysis of any gyrotron combining, in a natural sequence, simulation of beam formation in the electron-optical system and beam-field interaction in the resonant cavity.

The developed software package is a powerful tool for analysis, optimization and simulationbased design of gyrotrons. It has been validated successfully in the course of the development of a novel high harmonic gyrotron with axis-encircling electron orbits and a rare-earth permanent mag. nets [14].

We plan to continue the development and improvement of the physical models and the software tools for simulation of gyro-devices in order to apply them to: (i) optimization of the operational performance of the existing devices of Gyrotron FU series, (ii) interpretation of the data obtained from direct experiments and (iii) $\mathrm{CAD}$ of new tubes.

\section{Acknowledgements}

The results presented in this paper have been obtained in the framework of the collaboration between FIR FU Research Centre at Fukui University (Fukui, Japan), Shin-Etsu Chemical Co. (Takefu, Japan), Institute of Electronics of the Bulgarian Academy of Sciences (Soffa, Bulgaria) and the Institute of Applied Physics of the Russian Academy of Sciences (N. Novgorod, Russia). Part of the numerical calculations were made on $\mathrm{SX}-5$ computer at the Computer Center of the National Institute of Fusion Science (NTFS), Japan.

\section{References}

[1] Antonsen Jr. TM, Mondelli AA, Levush B, Verboncoeur JP, Birdsall CK. Proc IEEE 1999;87:804-38.

[2] Borie E. Int J Infrared Millimeter Waves 1986;7 1863-79.

[3] Borie E. Computation of radio-frequency behavior In: Edgcombe $\mathrm{CJ}$, editor. Gyrotron oscillators their principles and practice. London: Taylor\&Francis; 1993. p. $45-86$.

14] Boton M, Antonsen Ir. TM, Levush B, Nguyen K, Vlasov A. IEEE Trans Plasma Sci 1998;26:882-6.

[5] Gold SH, Fliflet AW. Int J Electron 1992;72:779-94.

[G] Sabchevski S, Mladenov G, Idehara T. Int J Infrared Millimeter Waves 1999;20:1019-35.

[7] Sabchevski S, Idehara T, Ogawa I, Glyavin M, Mitsudo S, Ohashi K, Kobayashi H. Int J Infrared Millimeter Waves 2000;21:1191-209.

[8] Sabchevski S, Mladenov G, Titov A, Barbarich I. Nucl Instr Meth 1996;A381:185-93.

[9] ELF Corp. Japan, Software ELF/MAGIC (Magnetic Field Analysis by Integral Computation), 1999.

[10] Flifet AW, Read ME, Chu KR, Seeley R. Int J Electron 1982:53:505-21.

[11] Sabchevski S, Idehara T, Ogawa I, Glyavin M, Obashi K. Int J Infrared Millimeter Waves 2002;23:675-92.

[12] Nusinovich GS. Int J Electron 1981;51:457.

[13] Dumbrajs $O$. Review of the theory of mode competition in gyrotrons. In: Edgcombe $\mathrm{CJ}$, editor. Gyrotron oscillators their principles and practice. London: Taylor \&Francis; 1993. p. 82-125.

[14] Idehara T, Ogawa I, Mitsudo S, Iwata $Y$, Watanabe S, Ohashi K, Kobayashi H, Yokoyama T, Zapevalov V, Glyavin M, Kuftin A, Malygin O, Sabchevski S. Development of a high harmonic gyrotron with an axisencircling electron beam and permanent magnet, Vacuum, this issue doi:10.1016/\%.racuum.2004.09.022 